\title{
Adjuvant chemotherapy in the patients with rectal cancer after neoadjuvant chemoradiotherapy and radical resection - YES
}

\author{
Rafał Stec
}

At the moment there are some scientific data supporting the use of adjuvant chemotherapy in patients with rectal cancer after neoadjuvant chemoradiotherapy and surgical treatment. The paper below presents some arguments for the use of adjuvant chemotherapy in the above clinical situation. The majority of deaths in patients with rectal cancer are caused by the presence of distant metastases, therefore there are significant grounds for the use of adjuvant chemotherapy in this group of patients. Summarising, there is no clear or scientific evidence against the use of adjuvant chemotherapy in patients with rectal cancer after preoperative chemoradiotherapy. In order to make a conclusive statement about the lack of any benefits from adjuvant therapy, it is necessary to carry out randomised studies on a homogeneous and standardised group of patients. Therefore, it seems appropriate to apply this therapy especially for patients with the $\mathrm{N}(+)$ features (with a tumour location between $10 \mathrm{~cm}$ and $15 \mathrm{~cm}$ ), similar to colon cancer cases.

NOWOTWORY J Oncol 2018; 68, 3: 152-156

Key words: rectal cancer, preoperative chemoradiotherapy, surgical treatment, adjuvant chemotherapy

Currently there is some scientific evidence supporting the use of adjuvant chemotherapy in patients with rectal cancer after neoadjuvant chemoradiotherapy and surgical treatment. The arguments for the usage of adjuvant therapy in such clinical conditions are presented below.

The majority of deaths in patients with rectal cancer are caused by the presence of distant metastases [1], therefore there are some essential grounds for the use of adjuvant chemotherapy in this group of patients. The task of adjuvant chemotherapy is to destroy micro-metastatic lesions, which remain invisible in imaging diagnostics.

Both surgical treatment and radiotherapy are solely the methods of local treatment, so their purpose is not the "liquidation" of the distant metastases. Fluoropyrimidines, in combination with radiotherapy, in neoadjuvant treatment are not used in doses which are systematically active (two courses are administered in the first and the fifth week of radiotherapy or three courses according to the de Gramont regimen as a consolidation therapy after a "short" radiotherapy) [1], that is why adjuvant chemotherapy should be used especially in patients with cancer dissemination risk factors, such as the presence of "positive" lymph nodes ( $\mathrm{N}+$ stage) or T4 stage.

A significant improvement in the efficiency of adjuvant chemotherapy in the third stage of colon cancer was obtained by adding oxaliplatin to the 2 -drug regimen (5-fluorouracil and folinic acid). In the "MOSAIC" trial (André et al. 2015) the 10-year OS (overall survival) was $67.1 \%$ for the patient group treated with the chemotherapy with oxaliplatin regimen (FOLFOX 4) compared to $59.0 \%$ for the group of patients treated solely with 5 -fluorouracil and folinic ac id) $(\mathrm{HR}=0.80 ; \mathrm{p}=0.016)$ [2]. We have to remember that this was a colon cancer whose treatment method differs significantly from that applied for rectal cancer, yet with the proper patient selection (the third disease stage) and with the use of the more effective chemotherapy regimen (based on oxaliplatin), a significant and desired objective can be achieved, i.e. the improvement of survival factors.

In the EORTC 22921 randomised trial (Bosset et al. 2014), 1011 patients with rectal cancer were randomly distributed 
into 4 groups: the first two were treated with preoperative radiotherapy or radio-chemotherapy, whilst the next two groups received adjuvant chemotherapy, after neoadjuvant therapy and after surgical intervention. No statistically significant improvement was obtained with regards to DFS (disease-free survival), or to OS; the 10-year DFS was $47.0 \%$ in the patient group treated with adjuvant chemotherapy as compared with $43.7 \%$ in the group of patients undergoing observation alone $(\mathrm{HR}=0.91 ; 95 \% \mathrm{Cl} 0.77-1.08 ; \mathrm{p}=$ 0.29), whilst the 10 -year OS was $51.8 \%$ as compared with $48.4 \%$ respectively ( $\mathrm{HR}=0.91 ; 95 \% \mathrm{Cl} 0.77-1.09, \mathrm{p}=0.32)$. It must be stressed that the evaluation of the efficiency of the applied adjuvant chemotherapy in patients with rectal cancer undergoing previously neoadjuvant radiotherapy or chemoradiotherapy, did not comprise the analysis of the subjects with $\mathrm{N}(+)$ stage (statistical analysis of the patients was based solely on the T stage), whereas this is precisely the group which might benefit most from the adjuvant treatment, like in the case of colon cancer patients; also here a suboptimal chemotherapy was used (without oxaliplatin), which is normally used in the adjuvant treatment of the colon cancer patients $[2,3]$. The lack of efficiency of the adjuvant chemotherapy applied after preoperative chemoradiotherapy and after surgical intervention was presented in another third phase randomised clinical trial (Aldo et al. 2014). The patients (655 patients) were randomly distributed to the two study arms: arm A comprised patients treated preoperatively with chemoradiotherapy and then under observation alone, whilst arm B were patients who additionally received 6 courses of adjuvant chemotherapy according to the regimen: 5 -fluorouracil with folinic acid. No significant differences between A and B groups were achieved with regards to the 5 -year DFS and 5-year OS: $62.8 \%$ vs $65.3 \%$ ( $p=08.82)$ and $70 \%$ vs $69.1 \%(p=0.772)$ respectively. In spite of the definite results of the study, a number of doubts linger: a significantly lower rate of patients in the $\mathrm{N}(+)$ stage were included in the study, but they are the most important target group for the use of adjuvant chemotherapy and, what is more these patients were included into the specific study arms in different manners: in arm A there were $24.5 \%$ patients, whereas in arm B 34.6\% (29.7\% of the total entire patient population). Some other doubts concern the lack of important clinical and pathomorphological data - in $13.2 \%$ patients, the $\mathrm{N}$ stage was marked as Nx. This would mean that in spite of a poorer prognosis in arm $B$ (a higher rate of patients in the $\mathrm{N}+$ stage) similar results in survival were obtained. The lack of significant improvement in both DFS and OS could also be the outcome of the application of a suboptimal chemotherapy regimen: 5 -fluorouracil with folinic acid (without oxaliplatin) [4].

Another third-phase randomised clinical trial, prepared by the Dutch group, "PROCTOR-SCRIPT" (Breugom et al. 2015), in which 437 patients were included (221 subjects in the observation group and 216 patients treated with adjuvant chemotherapy), also failed to confirm the efficiency of adjuvant chemotherapy. The 5-year OS for the group undergoing observation alone was $79.2 \%$ vs $80.4 \%$ in the group treated with adjuvant chemotherapy $(\mathrm{HR}=0.93 ; 95 \%$ $\mathrm{Cl} 0.62-1.39 ; \mathrm{p}=0.73$; HR for DFS was $0.80 ; 95 \% \mathrm{Cl} 0.60-1.07$; $p=0,13)$, whilst the 5 -year cumulative frequency of loco-regional relapse was $7.8 \%$ for both groups and the 5 -year cumulative frequency of distant metastases was $38.5 \%$ vs $34.7 \%$ respectively $(p=0.39)$. Nevertheless, in the analysis of the patient characteristics, it must be emphasised that the rate of patients who received neoadjuvant chemotherapy before the adjuvant chemotherapy was only $14.0 \%$ for both groups $(12.7 \%$ in the group with observation alone and $15.3 \%$ in the group treated with adjuvant chemotherapy), which makes it impossible to draw reliable conclusions in this group of patients. Moreover, chemotherapy was based on 5-fluorouracil or capecitabin, without oxaliplatin [5].

The benefit in the use of adjuvant chemotherapy (Hong et al. 2014) was obtained in the second-phase clinical trial, "ADORE" ("ADjuvant Oxaliplatin in REctal cancer"). The study comprised 321 rectal cancer patients in the second and third stage (ypT3-4N0 or any ypT, N1-2 in screening) after neoadjuvant chemotherapy. Adjuvant chemotherapy consisted of a 2-drug regimen: 5-fluorouracil with folinic acid or the FOLFOX regimen in the second group. Some improvement with regards to the 3-year DFS was obtained for the patient group receiving chemotherapy based on the FOLFOX regimen in comparison with the patient group treated with 5-fluorouracil with folinic acid (71.6\% vs $62.9 \%$; $\mathrm{HR}=0.657$; 95\% Cl 0.434-0.994; $p=0.047)$. Some particular benefit with regards to the 3-year DFS was obtained in the group of patients with the third stage disease in comparison with patients with the second stage $(66.6 \%$ vs $57.3 \%$; $\mathrm{HR}=0.602$; $95 \% \mathrm{Cl} 0.371-0.977 ; \mathrm{p}=0.040)$ and $(81.6 \%$ vs $71.3 \%$; $\mathrm{HR}=$ $0,744,95 \% \mathrm{Cl} 0,334-1,657 ; \mathrm{p}=0,47$ ) respectively. The 3-year OS also turned out to be better in the group of patients receiving the chemotherapy regimen based on oxaliplatin in comparison with the group of patients treated with the 2 -drug regimen $(95.0 \%$ vs $85.7 \%$; $\mathrm{HR}=0.456,95 \% \mathrm{Cl}$ $0.215-0.970 ; p=0.036$ ). Summing up this study, it must be stressed that the largest benefit in adjuvant treatment was gained by the patients with the third stage disease, which correlates with the results of adjuvant treatment in colon cancer [6].

The metanalysis which shows the lack of any benefit in such a course of treatment is that which is presented by Breugom et al. in 2015, which comprised 4 third-phase randomised clinical trials (1198 rectal cancer patient in total: “I-CNR-RT",“PROCTOR-SCRIPT”, “EORTC 2292”,'“CHRONICLE”), in which adjuvant treatment consisted in a chemotherapy regimen based on 5-fluorouracil ( 3 trials), capecitabine (1 study) or XELOX regimen (1 study). No significant benefit was 
shown in the entire patient population with regards to the OS with the use of adjuvant chemotherapy after a preoperative neoadjuvant chemoradiotherapy $(\mathrm{HR}=0.97 ; 95 \% \mathrm{Cl}$ $0.81-1.17 ; p=0.775)$. However, in the subgroup analysis, a significant improvement in DFS and remote recurrence was obtained in patients with the location of the primary tumour between $10 \mathrm{~cm}$ and $15 \mathrm{~cm}$ from the edge of the anus (HR $=0.59 ; 95 \% \mathrm{Cl} 0.40-0.85 ; \mathrm{p}=0.005$ and $\mathrm{HR}=0.61,95 \% \mathrm{Cl}$ $0.40-0.94 ; p=0.025$ respectively). In the discussion of the metanalysis, the heterogenicity (the lack of homogeneity of the results) of the studies qualified for evaluation. In spite of the lack of proof with regards to significant differences to the heterogenicity of the qualified studies, some significant incompliances concerned, among others, different chemotherapy regimens applied in the adjuvant treatment, various regimens of preoperative therapy, taking into consideration the rate of the patients who were undergoing radiotherapy alone (2 studies), the diverse moment of the randomisation of the patients (randomisation before or after surgical treatment) or different standards of surgical intervention (total mesorectal excision and radical surgical intervention). Taking into consideration the doubts presented above, one cannot draw definite conclusions concerning the lack of efficiency of adjuvant chemotherapy in rectal cancer patients after preoperative treatment combined with chemoradiotherapy [7].

In another metanalysis (5 randomised clinical trials, 2398 patients), there was no statistically significant benefit in the use of adjuvant chemotherapy in comparison with the group undergoing observation alone among the rectal cancer patients after preoperative radiotherapy or radio-chemotherapy (Bujko et al. 2015). The differences both in OS and DFS were not statistically significant between the group with chemotherapy and the group of patients without adjuvant treatment $(\mathrm{HR}=0.95 ; 95 \% \mathrm{Cl} 0.82-1.10 ; \mathrm{p}=0.49$ and $\mathrm{HR}=0.92 ; 95 \% \mathrm{Cl} 0.80-1.04 ; \mathrm{p}=0.19$ ) respectively. Also no statistically significant difference was obtained with regards to DFS (4 randomised clinical trials, 2710 patients) between the group on chemotherapy based on oxaliplatin and the group of patients without oxaliplatin $(\mathrm{HR}=0.84$, $95 \% \mathrm{Cl} 0.66-1.06 ; \mathrm{p}=0.15)$ in spite of the fact that in two of four analysed trials there was a statistically significant difference in DFS to the advantage of the patients treated in chemotherapy with oxaliplatin.

Similarly as in the case of other clinical trials or metanalyses, a number of doubts arise, concerning the limited population of patients included into specific clinical trials, which makes it impossible to catch differences smaller than $3-5 \%$ with respect to the 5 -year OS (type II error), suboptimal methodology (e.g. randomisation before preoperative treatment which decreases the possibilities of observing the effect of post-operative chemotherapy), diverse patient groups included into the trials and metanalyses (e.g. pre- operative radiotherapy, varied disease stages without $\mathrm{N}+$ a stage or even ypTON0 stage or the first stage of disease), and also the fact in 3 of 9 studies in the metanalysis, only abstracts were available (no reviews, which always present the discussion of the obtained results) [8].

In a systematic review and metanalysis (in total 4 randomised clinical trials: "CAO/ARO/ AIO-04","PETACC-6","ADORE" and "CHRONICLE") published by Zhao L et al. (2016), the final analysis concerned 2793 rectal cancer patients in the second or third disease stage, who, after preliminary treatment with chemoradiotherapy followed by a surgical intervention, received adjuvant treatment consisting in chemotherapy regimens composed of capecitabine/5-fluorouracil/5-fluorouracil with folinic acid or underwent observation alone (one group) or chemotherapy regimens containing oxaliplatin in connection with 5-fluorouracil and folinic acid or capecitabine (the second group). In the group of patients treated according to chemotherapy regimens containing oxaliplatin, in comparison with the chemotherapy regimens without this drug, a statistically significant prolongation of DFS as the primary endpoint was obtained ( $\mathrm{HR}=0.85 ; 95 \%$ $\mathrm{Cl} 0.73-0.98 ; \mathrm{p}=0.03$ ), but no OS prolongation was obtained - and this was the secondary study endpoint ( $\mathrm{HR}=0.64$; $95 \% \mathrm{Cl} 0.35-1.17 ; \mathrm{p}=0.15)$. In the presented metanalysis, the qualified studies differed significantly from each other, among others by the chemotherapy regimens (oxaliplatin was used in preoperative treatment only in two studies) and the planned recruitment was not completed ("CHRONICLE" study). Moreover, in three trials, adjuvant chemotherapy comprised patients with complete pathological remission after the ypCR treatment (ypTONOM0) or the first disease stage (the group of patients who do not benefit from adjuvant therapy). In three trials, the rate of patients who completed the planned treatment in total or who received the majority of the chemotherapy courses totalled $43 \%$ to $55 \%$ (the group of patients with adjuvant treatment of chemotherapy based on 5-fluorouracil) and, most importantly, the group of patients solely with the third pathological disease stage was not evaluated separately whist this is the group of patients who may benefit from such a course of treatment the most. Similarly, as in the previous metanalysis, the differences and doubts shown by the authors do not allow for any definite conclusions [9]. The summary of the discussed studies and metanalyses is presented in Table I.

In conclusion, as of the current moment, there is no definite scientific evidence providing grounds for the use of adjuvant chemotherapy in rectal cancer patients after preoperative chemoradiotherapy. It is necessary to carry out a randomised study, yet it should concern a homogenous and standardised group of patients with respect to their treatment in order to determine definite opinions concerning the lack of benefits in adjuvant therapy. That is why currently, such therapy seems to be justified especially in 
Table I. The presentation of the studies and metanalyses

\begin{tabular}{|c|c|c|c|c|}
\hline Author/year & $\begin{array}{l}\text { The number of } \\
\text { subjects }\end{array}$ & Type of study & DFS improvement & OS improvement \\
\hline $\begin{array}{l}\text { Bosset et al. } 2014 \\
\text { "EORTC 22921" }\end{array}$ & 1011 & The third-phase randomised clinical study & NO & NO \\
\hline Aldo et al. 2014 & 655 & The third-phase randomised clinical study & NO & NO \\
\hline $\begin{array}{l}\text { Breugom et al. } 2015 \\
\text { "PROCTOR-SCRIPT" }\end{array}$ & 437 & The third-phase randomised clinical study & NO & NO \\
\hline $\begin{array}{l}\text { Hong et. al. } 2014 \\
\text { "ADORE" }\end{array}$ & 321 & The second-phase randomised clinical study & YES & YES \\
\hline Breugom et al. 2015 & 1198 & $\begin{array}{l}\text { The metanalysis of } 4 \text { studies: } \\
\text { "I-CNR-RT","“PROCTOR-SCRIPT", “EORTC 2292", } \\
\text { CHRONICLE" }\end{array}$ & $\begin{array}{l}\text { YES } \\
\text { With the primary } \\
\text { tumour location } \\
\text { between } 10 \text { and } 15 \mathrm{~cm}\end{array}$ & NO \\
\hline \multirow[t]{3}{*}{ Bujko et al. 2015} & 2398 & $\begin{array}{l}\text { The metanalysis of } 5 \text { studies: } \\
\text { "EORTC 22921","Italian trial"," "PROCTOR/ } \\
\text { SCRIPT", "CHRONICLE", "QUASAR" }\end{array}$ & NO & NO \\
\hline & & $\begin{array}{l}\text { The metanalysis of } 4 \text { studies: } \\
\text { "PETACC-6","CAO/ARO/AIO-04", "ADORE”, } \\
\text { "ECOG E3201" }\end{array}$ & & \\
\hline & 2710 & & NO & -------- \\
\hline Zhao L et al. 2016 & 2973 & $\begin{array}{l}\text { The metanalysis of } 4 \text { studies: } \\
\text { "CAO/ARO/ AIO-04", "PETACC-6", "ADORE”, } \\
\text { "CHRONICLE" }\end{array}$ & YES & NO \\
\hline
\end{tabular}

DFS - disease-free survival, OS - overall survival

the group of patients with the $\mathrm{N}(+)$ stage (at least with the tumour location between $10 \mathrm{~cm}$ and $15 \mathrm{~cm}$ ), similar to colon cancer; especially as there are no significant differences between colon cancer and rectal cancer as for their genetics and response to the palliative treatment [8].

\section{Response}

In the agreed recommendations concerning the controversies in the primary treatment of rectal cancer, $77 \%$ of "panellists" opted for the use of adjuvant chemotherapy in the case of confirmed "positive" lymph nodes after neoadjuvant chemotherapy (ypN+). To sum up this subchapter of these recommendations concerning adjuvant treatment, the authors pointed to the use of adjuvant chemotherapy which should be considered the standard treatment in rectal cancer patients with the tumour location between $10 \mathrm{~cm}$ and $15 \mathrm{~cm}$ from the edge of the anus, with the presence of "involved" lymph nodes before neoadjuvant treatment ( $\mathrm{cN}+$ ) or after its completion ( $\mathrm{ypN}+$ ), and the chemotherapy regimen should contain oxaliplatin (47\% votes for "yes", $16 \%$ for "no", 37\% abstentions [1].

A similar sentiment was also seen in the most recent ESMO guidelines concerning the treatment of rectal cancer (Glynne-Jones et al. 2017), whose authors recommend considering the use of adjuvant treatment with chemotherapy in the third and second pathological disease stage ("yp") with the presence of risk factors ("high-risk" group) after preoperative radiotherapy or chemoradiotherapy [10].
Also the NCCN recommendations (The National Comprehensive Cancer Network, version 4.2017) are definite with regards to the indications connected with the use of adjuvant chemotherapy after neoadjuvant chemotherapy. In patients with the T3N0 or ( $\mathrm{N}+$ ) or T4 stages and/or a non resectable tumour, after the neoadjuvant treatment with radiotherapy or chemoradiotherapy and surgical intervention, it is recommended to use adjuvant chemotherapy based on oxaliplatin (regimens such as: FOLFOX or CAPOX, or 5-fluorouracil with folinic acid or capecitabine), and the entire perioperative systemic treatment should last 6 months in total [11].

Conflict of interest: none declared

\section{Rafał Stec, MD, PhD}

Medical University of Warsaw

Department of Oncology

ul. Stępińska 19/25

00-739 Warszawa, Poland

e-mail:drrafals@wp.pl

Received \& Accepted: 8 Apr 2018

Based on presentation at the VI Annual Conference of the Nowotwory Journal of Oncology, 'Oncological Debates, held in Warszawa, 6-7th April 2018 
References (the most significant publications)

1. Lutz MP, Zalcberg JR, Glynne-Jones R et al. Second St. Gallen European Organisation for Research and Treatment of Cancer Gastrointestinal Cancer Conference: consensus recommendations on controversial issues in the primary treatment of rectal cancer. Eur J Cancer 2016; 63:11-24.

2. André T, de Gramont A, Vernerey D et al. Adjuvant fluorouracil, leucovorin, and oxaliplatin in stage II to III colon cancer: updated 10-year survival and outcomes according to braf mutation and mismatch repair status of the MOSAIC study. J Clin Oncol 2015; 33: 4176-4187.

3. Bosset JF, Calais G, Mineur L et al. Fluorouracil-based adjuvant chemotherapy after preoperative chemoradiotherapy in rectal cancer: long-term results of the EORTC 22921 randomised study. Lancet Oncol 2014; 15: 184-190.

4. Sainato A, Cernusco Luna Nunzia V, Valentini V et al. No benefit of adjuvant fluorouracil leucovorin chemotherapy after neoadjuvant chemoradiotherapy in locally advanced cancer of the rectum (LARC): Long term results of a randomized trial (I-CNR-RT). Radiother Oncol 2014; 113: 223-229.

5. Breugom AJ, van Gijn W, Muller EW et al. Adjuvant chemotherapy for rectal cancer patients treated with preoperative (chemo)radiotherapy and total mesorectal excision: a Dutch Colorectal Cancer Group (DCCG) randomised phase III trial. Ann Oncol 2015; 26: 696-701.
6. Hong YS, Nam BH, Kim KP et al. Oxaliplatin, fluorouracil, and leucovorin versus fluorouracil and leucovorin as adjuvant chemotherapy for locally advanced rectal cancer after preoperative chemoradiotherapy (ADORE): an open-label, multicentre, phase 2, randomised controlled trial. Lancet Oncol 2014; 15: 1245-1253.

7. Breugom AJ, Swets M, Bosset JF et al. Adjuvant chemotherapy after preoperative (chemo)radiotherapy and surgery for patients with rectal cancer: a systematic review and meta-analysis of individual patient data. Lancet Oncol 2015; 16: 200-207.

8. Bujko K, Glimelius B, Valentini V. Postoperative chemotherapy in patients with rectal cancer receiving preoperative radio(chemo)therapy: A meta-analysis of randomized trials comparing surgery +/-fluoropyrimidine and surgery + a fluoropyrimidine $+/$ - oxaliplatin. Eur J Surg Oncol 2015; 41: 713-723.

9. Zhao L, Liu R, Zhang Z et al. Oxaliplatin/fluorouracil-based adjuvant chemotherapy for locally advanced rectal cancer after neoadiuvant chemoradiotherapy and surgery: a systematic review and meta-analysis of randomized controlled trials. Colorectal Dis 2016; 18: 763-772.

10. Glynne-Jones R, Wyrwicz L, Tiret E et al. Rectal cancer: ESMO Clinical Practice Guidelines for diagnosis, treatment and follow-up. Ann Oncol 2017; 28 (Suppl 4): iv22-iv40.

11. NCCN Clinical Practice Guidelines in Oncology: NCCN Guidelines $^{\circ}$ Rectal Cancer Version 4.2017 — January 18, 2018; NCCN.org. 